\title{
Additive and Interactive Effects of Stimulus Degradation: No Challenge for $\mathrm{CDP}+$
}

\author{
Johannes C. Ziegler \\ Aix-Marseille Université and Centre National de la Recherche \\ Scientifique
}

\author{
Conrad Perry \\ Swinburne University of Technology
}

\author{
Marco Zorzi \\ Università di Padova
}

\begin{abstract}
S. O'Malley and D. Besner (2008) showed that additive effects of stimulus degradation and word frequency in reading aloud occur in the presence of nonwords but not in pure word lists. They argued that this dissociation presents a major challenge to interactive computational models of reading aloud and claimed that no currently implemented model is able to simulate additive effects in these conditions. In the current article, it is shown that the connectionist dual process model (CDP+) can simulate these effects because its nonlexical route is thresholded. The authors present a series of simulations showing that $\mathrm{CDP}+$ can not only simulate the precise dissociation observed by O'Malley and Besner but more generally can produce additive effects for a wide range of parameter combinations and different sets of items. The nonlexical route of CDP+ was not modified post hoc to deal with the effects of stimulus quality, but it had been thresholded for principled reasons before it was known that these effects existed. Together, the effects of stimulus quality on word frequency do not challenge $\mathrm{CDP}+$ but rather provide unexpected support for its architecture and processing dynamics.
\end{abstract}

Keywords: modeling, reading, word recognition, stimulus quality, additive effects

Supplemental materials: http://dx.doi.org/1037/a0013738.supp

The effects of stimulus degradation on variables, such as frequency and nonword length, provide important information about the architecture and processing dynamics of visual word recognition (Besner \& Roberts, 2003; Borowsky \& Besner, 1993). Most studies of reading aloud have found overadditive effects of stimulus degradation and word frequency (O'Malley, Reynolds, \& Besner, 2007; Yap \& Balota, 2007) but additive effects of stimulus degradation on nonword length (Besner \& Roberts, 2003). O'Malley and Besner (2008) solved this seemingly contradictory pattern by demonstrating that additive effects come and go with the presence or absence of nonwords. Indeed, they showed that mixing words and nonwords when reading aloud produces additive effects of stimulus quality and word frequency, whereas presenting the same words without nonwords produces overadditive effects.

O'Malley and Besner (2008) argued that this pattern provides a major challenge to current computational models, as apparently no

Johannes C. Ziegler, Aix-Marseille Université and Centre National de la Recherche Scientifique, Marseille, France; Conrad Perry, Faculty of Life and Social Sciences, Swinburne University of Technology, Melbourne, Victoria, Australia; Marco Zorzi, Dipartimento di Psicologia Generale and Center for Cognitive Science, Università di Padova, Padua, Italy.

Marco Zorzi was supported by European Research Council Grant 210922-GENMOD.

Correspondence concerning this article should addressed to Johannes C. Ziegler, Department of Cognitive Psychology, Aix-Marseille Université, 3 place Victor Hugo 13331, Marseille, Cedex 3, France. E-mail: Johannes .Ziegler@univ-provence.fr computational model of reading can simulate this effect. They stated, "We are aware of no implemented model of visual word recognition in its current form that produces additivity of stimulus quality and word frequency in reading aloud" (p. xxx). O'Malley and Besner proposed that the dual route cascaded (DRC) model (Coltheart, Rastle, Perry, Langdon, \& Ziegler, 2001) could be modified to produce additive effects by thresholding the letter level, but such modification would mean that DRC would always produce additive effects of stimulus quality and word frequency. This would not be correct, because additive effects are obtained only in the presence of nonwords. Thus, at first sight, effects of stimulus quality, which vary as a function of the presence of nonwords, appear to seriously challenge current computational models. Our aim in this article is to take on that challenge by showing that $\mathrm{CDP}+$, the new version of the connectionist dual process model (Perry, Ziegler, \& Zorzi, 2007; Zorzi, Houghton, \& Butterworth, 1998) can easily capture the complex pattern of additive and interactive effects of stimulus degradation and frequency.

\section{Cascaded and Thresholded Processes in CDP+}

The finding that stimulus degradation has additive effects on variables, such as frequency or nonword length, has been taken to suggest that letter activation is likely to be thresholded rather than cascaded (e.g., Besner \& Roberts, 2003; Reynolds \& Besner, 2004). If the letter level is thresholded, this means that letters need to be fully activated before they send activation to subsequent processing levels. If letter activation were thresholded, stimulus 
degradation would affect only the rise of activation in the letter units and not the subsequent processing stages. As a consequence, one would predict, stimulus quality would have an additive effect on variables beyond the letter level.

In CDP+ (Perry et al., 2007), there is both thresholded and cascaded processing between the letter level and subsequent processing levels. On the lexical route, activation between letter and word units is cascaded (i.e., letter units send their activation continuously to the corresponding word units). On the sublexical route, however, activation between letter units and grapheme units is thresholded (i.e., letters become available to the graphemic buffer only when their activation is above a given threshold). The assumption behind this choice is that the grapheme parser needs to select a single correct letter from the many that are potentially available in each letter position and that this selection can occur only when robust information about letter identity is available. Once a single letter has been selected, it is assumed, the grapheme parser treats the selected letter as fully active, even if it is not at the letter level.

As noted by Perry et al. (2007), the fact that letter activation on the sublexical route is thresholded in CDP+ leads to the natural prediction that the effects between stimulus quality and variables that affect sublexical processing should be additive. For example, when the model reads nonwords, $\mathrm{CDP}+$ should produce a perfectly additive effect of stimulus quality and nonword length.

\section{Additive Effects of Stimulus Quality and Nonword Length}

To prove our claim that $\mathrm{CDP}+$ produces perfectly additive effects of stimulus quality and nonword length when the only parameter that is changed simply degrades the visual input, we ran the nonword stimuli of Besner and Roberts (2003) through the $\mathrm{CDP}+$ model. We manipulated stimulus degradation parametrically by reducing feature-to-letter activation from .005 (original value) to $.004, .003, .002$, and .001 . Reducing the value of featureto-letter activation has the effect of slowing the activation buildup at the letter level, and it has been used in previous modeling studies to simulate stimulus degradation (Besner \& Roberts, 2003; Reynolds \& Besner, 2004).

The results of this simulation are presented in Figure 1. It is clear that $\mathrm{CDP}+$ produces a perfectly additive effect of stimulus quality and nonword length across a wide range of degradations (all $F$ s for the interaction between stimulus quality and length were smaller than 1). Note that these simulations were based on the manipulation of a single parameter (i.e., feature-to-letter activation).

\section{Additive Versus Interactive Effects of Stimulus Quality and Frequency}

Of course, the critical effect in O'Malley and Besner (2008) was not nonword length but additive effects of stimulus quality and word frequency in the presence of nonwords. We argue that CDP+ can simulate these effects if we assume that adding nonwords biases the system to deemphasize lexical processing in favor of a greater reliance on the nonlexical route. One reason for the system to deemphasize lexical processing, as suggested by O'Malley and Besner, is to avoid lexical captures, (i.e., trials in which the

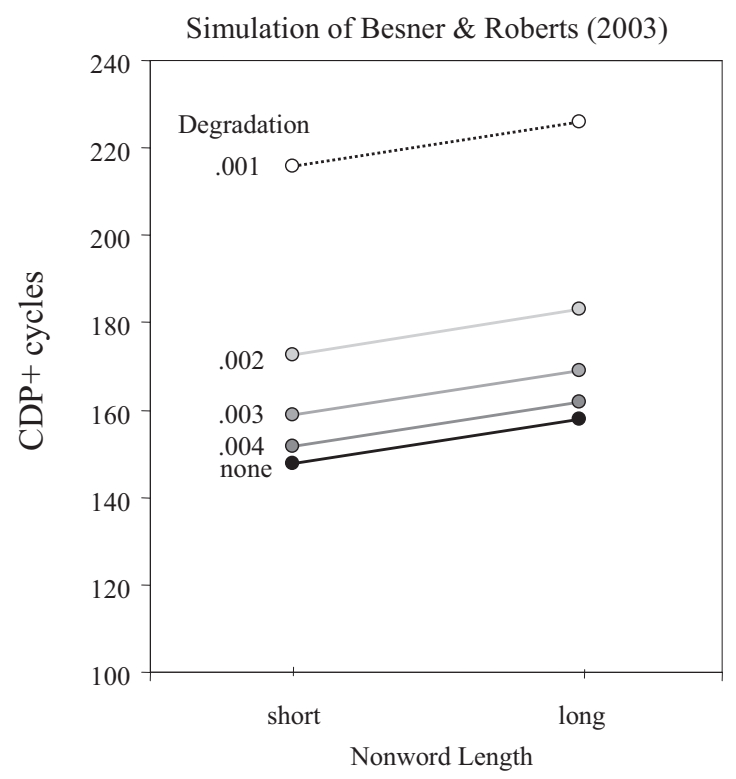

Figure 1. $\mathrm{CDP}+$ simulation of the additive effect of stimulus degradation and nonword length as observed by Besner and Roberts (2003). Stimulus degradation was simulated by reducing feature-to-letter activation from .005 (original value, no degradation) to $.004, .003, .002$ and $.001 . \mathrm{CDP}+=$ connectionist dual process model.

network gives a "word" response to a nonword). More generally, a number of studies have suggested that skilled readers strategically adjust the degree to which lexical and sublexical information contribute to reading aloud in the presence of nonwords or irregular words (Reynolds \& Besner, 2008; Zevin \& Balota, 2000). In line with these findings, we assume that adding nonwords to a reading aloud experiment changes the balance between lexical and nonlexical processing. Because the nonlexical route is thresholded in $\mathrm{CDP}+$ (for principled reasons and not post hoc to simulate effects of stimulus quality; see below for discussion), we argue that greater reliance on the nonlexical route will naturally produce additive effects under certain conditions.

In their 2008 article, O'Malley and Besner questioned whether $\mathrm{CDP}+$ could account for their findings and called for a demonstration that CDP + can simulate the results. Such a demonstration is provided below. We carried out four simulations using the monosyllabic items of O'Malley and Besner's Experiment 3 (68 high-frequency and 69 low-frequency words; see Appendix A online in the supplemental materials). Experiment 3 was of primary interest because it was the only experiment that explicitly compared word only (pure) and word/nonword (mixed) conditions. The word-only condition produced an overadditive effect, whereas the mixed condition produced an additive effect between stimulus quality and frequency.

Stimulus degradation was simulated by reducing the feature-toletter activation parameter. This parameter was reduced until the model produced a ratio between the size of the degradation effect and the size of the frequency effect that was comparable with the human data (.001 in the present simulation). The shift from lexical to nonlexical processing was simulated by reducing the activation of the lexical route via reduction of letter-to-word activation. 
Because there is a fine balance between the lexical and the nonlexical route, reducing the activation of the lexical route automatically gives greater weight to the nonlexical route. In summary, the clear word-only condition was simulated with the original CDP+ model. The degraded word-only condition was simulated by reducing feature-to-letter activation. The clear mixed condition was simulated by reducing letter-to-word activation. Finally, the degraded mixed condition was simulated by reducing feature-toletter and letter-to-word activation. A summary of these changes and the precise parameter values can be found in Table 1 .

The results of the simulation are presented in Figure 2 next to the human data from O'Malley and Besner (2008). Model errors were excluded from the latency analysis $(4.8 \%)$. The data from one $3 S D$ outlier (could) was also excluded. All items are presented in Appendix A in the supplemental materials along with the item latencies and errors for all conditions. As for the human data, the model made more errors in the degraded than in the clear condition ( $7.7 \%$ vs. $1.8 \%$, respectively). The latency data from the simulations were submitted to an analysis of variance (ANOVA), with frequency (high vs. low), condition (pure vs. mixed), and stimulus quality (clear vs. degraded) as factors. Condition and stimulus quality were within-item factors, and frequency was a betweenitems factor.

As for the human data, there was a significant main effect of frequency, $F(1,121)=113.3, p<.0001$; condition, $F(1,121)=$ $600.7, p<.0001$; and stimulus quality, $F(1,121)=6,068.4, p<$ .0001 . The condition effect reflected the finding that latencies were slower in the mixed condition ( 147.5 cycles) than in the word-only condition (137.4 cycles). Most important, the triple interaction was highly significant, $F(1,121)=208.1, p<.0001$. To further assess this triple interaction, we conducted two separate $2 \times 2$ ANOVAs, with frequency and stimulus quality as factors for pure and mixed conditions. In the pure condition, there was a significant interaction between the effects of stimulus quality and frequency, $F(1$, $125)=121.7, p<.0001$. In the mixed condition, however, the interaction between the effects of frequency and stimulus quality was not significant, $F(1,121)=2.2, p=.14$; this nonsignificance reflected the fact that the effects of stimulus quality and frequency were additive in the mixed condition.

To verify whether the present parameter set would allow $\mathrm{CDP}+$ to simulate the additive effects of stimulus quality and frequency reported in Experiments 1 and 2 of O'Malley and Besner (2008), we confronted the model with the items of Experiments 1 and 2. As in Experiment 3, O'Malley and Besner's goal in these two experiments was to show the existence of an additive effect of stimulus quality and frequency in

Table 1

Parameters Used in Simulating the Effects of Stimulus Quality and Word Frequency for Experiment 3 of O'Malley and Besner (2008)

\begin{tabular}{llcc}
\hline Condition & Stimulus quality & $\begin{array}{c}\text { Feature-to-letter } \\
\text { activation }\end{array}$ & $\begin{array}{c}\text { Letter-to-word } \\
\text { activation }\end{array}$ \\
\hline Word only & clear & .005 (original) & .0750 (original) \\
Mixed & $\begin{array}{l}\text { degraded } \\
\text { clear }\end{array}$ & .001 & .0750 (original) \\
& degraded & .005 (original) & .0598 \\
& & .001 & .0598 \\
\hline
\end{tabular}

the presence of nonwords. Both experiments used the same set of items, but these items were completely different from the ones used in Experiment 3. Thus, if CDP + were to simulate an additive pattern of stimulus quality and frequency with this new set of items, this result would certainly be strong evidence for the generality and robustness of the model's behavior.

We therefore used CDP + to obtain simulations for the monosyllabic items of Experiments 1 and 2 (64 low-frequency and 68 high-frequency words). The results are presented in Figure 3 next to the human data (all items and item means can be found in Appendix B in the supplemental materials). Model errors were excluded from the latency analysis $(3.0 \%)$. The model made more errors in the degraded than in the clear condition $(5.3 \%$ vs. $0.8 \%$, respectively). The latency data were submitted to a $2 \times 2$ repeatedmeasures ANOVA, with frequency and stimulus quality as factors. The results showed a main effect of frequency, $F(1,123)=80.1$, $p<.0001$, and stimulus quality, $F(1,123)=6207.7, p<.0001$ The effects of stimulus quality and frequency were additive, as confirmed by a nonsignificant interaction between the effects of stimulus quality and frequency, $F(1,123)=2.2, p=.15$.

Of course, it could be argued that one can always find a "lucky" parameter combination that would produce the desired additive effect but that the effect would not generalize to a wider set of parameter combinations. This issue was explored with parametric simulations, in which we looked at all possible parameter combinations within our parameter space. That is, we crossed four degradation levels $(.004, .003, .002, .001)$ with six letter-to-word activation levels $(.070, .065, .060, .055, .050, .045)$. For each of the 24 parameter combinations, we produced a $\mathrm{CDP}+$ simulation using a small set of low- and high-frequency words (10 items per group) from the O'Malley and Besner (2008) study. We also conducted six "nondegraded" simulations for the six levels of letter-to-word activation. Each of the 24 models that spanned the entire parameter space was assessed in an ANOVA, with degradation (clear vs. degraded) and word frequency (high vs. low) as factors. This analysis allowed us to test which of the 24 parameter combinations would produce an additive or over/underadditive pattern of stimulus quality and word frequency. The results are presented in Figure 4. This figure presents a density map of $F$ values for the interaction between stimulus quality and word frequency on a semilogarithmic scale. The density map has been smoothed with two-dimensional data interpolation. $F$ values smaller than about 4 are associated with an additive pattern, whereas $F$ values greater than 4 are associated with an over/ underadditive pattern.

As can be seen in Figure 4, when letter-to-word activation was normal (i.e., .075) or slightly reduced (.070), CDP+ indeed produced an overadditive pattern for all levels of stimulus degradation (bottom left corner). This pattern is consistent with the word-only data of O'Malley and Besner (2008). When letter-to-word activation was further reduced (simulating word/ nonword mixing), the pattern became additive. Indeed, there is a fairly large region of parameter combinations that produce perfect additivity (blue region, in which $F<1$ ), and this region clearly shows that $\mathrm{CDP}+$ can produce an additive pattern across a wide range of parameter combinations. Finally, the model produced an underadditive pattern of stimulus quality and word frequency for severe degradation combined with strongly reduced lexical influence. 
Human Data (Exp 3)

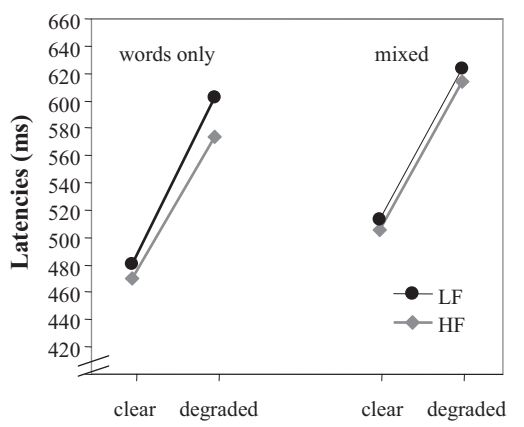

$\mathrm{CDP}+$ Simulation $(\operatorname{Exp} 3)$

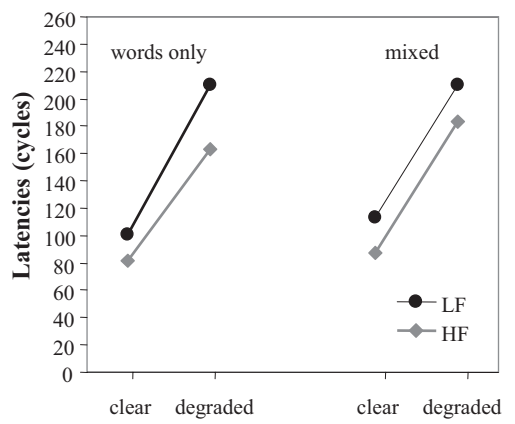

Figure 2. $\mathrm{CDP}+$ simulation of the effects of stimulus quality and word frequency for pure and mixed lists (O'Malley \& Besner, 2008, Experiment 3) with the parameter values specified in Table 1. CDP $+=$ connectionist dual process model; $\mathrm{LF}=$ low frequency; $\mathrm{HF}=$ high frequency.

\section{Discussion}

In the this article, we have shown that $\mathrm{CDP}+$ can simulate both an overadditive effect in normal (i.e., word-only) conditions and an additive effect in mixed (i.e., word/nonword) conditions. When we reduced stimulus quality by simply reducing feature-to-letter activation, the original $\mathrm{CDP}+$ produced an overadditive effect of stimulus quality and word frequency, as observed in the human data. However, the addition of nonwords to the experiment changed the pattern from overadditivity to additivity. CDP + is able to simulate this shift by assuming that the addition of nonwords has the effect of deemphasizing the lexical activation (possibly to avoid lexical captures as suggested by O'Malley \& Besner, 2008) and thus of giving greater weight to the nonlexical route. When the lexical activation is reduced and input is degraded, $\mathrm{CDP}+$ can simulate a perfectly additive pattern across a wide range of parameter combinations and different sets of items, as demonstrated in the present simulations. This demonstration clearly shows that at least one implemented computational model, $\mathrm{CDP}+$, can produce additive effects of stimulus quality and frequency.

Despite the rather striking fits between the model and the human data, there is one small discrepancy that needs to be discussed. In the human data of Experiment 3 (see Figure 2), the size of the frequency effect in the clear conditions was not affected by the addition of nonwords. Indeed, O'Malley and Besner (2008) reported a nonsignificant interaction when the frequency effect in the pure condition was compared with that in the mixed condition. However, when the same analysis was done on the model latencies, there was a small but significant interaction reflective of the fact that the simulations exhibited a slightly larger frequency effect in the mixed than in the pure condition ( 24 vs. 19 cycles, respectively). Although it is small, this is a real effect because reducing letter-word activation (to simulate the strategic shift related to the addition of nonwords) penalizes low-frequency words more than high-frequency words and thus produces greater frequency effects in the mixed than in the pure condition. This finding is somewhat counterintuitive, because one would have expected that greater reliance on nonlexical processing would have reduced the frequency effect. Indeed, speeding up the nonlexical route would certainly reduce the frequency effect and produce additive effects of stimulus quality, as shown before. However, there are two problems with this solution: The frequency effect was not reduced in the human data, and speeding up the nonlexical route would also speed up overall latencies in the mixed condition, which would be inconsistent with the human data.

In anticipation of this discrepancy being put forward as the major criticism of the present simulations, we make two points. First, the increase in the size of the frequency effect in the clear conditions is very small, and it probably is significant only because the statistical test is very sensitive (i.e., word/nonword mixing was manipulated within items in the model but between subjects in the human data). Second, we made the choice to simulate strategic shifts by changing a single parameter. However, the literature on route emphasis and pathway control reveals that it is quite clear that adaptive changes in response to nonwords (or irregular words) are fairly complex (e.g., Kinoshita \& Lupker, 2007; Reynolds \& Besner, 2008; Zevin \& Balota, 2000). Thus, there are some details about strategic shifts that are probably not captured in the present

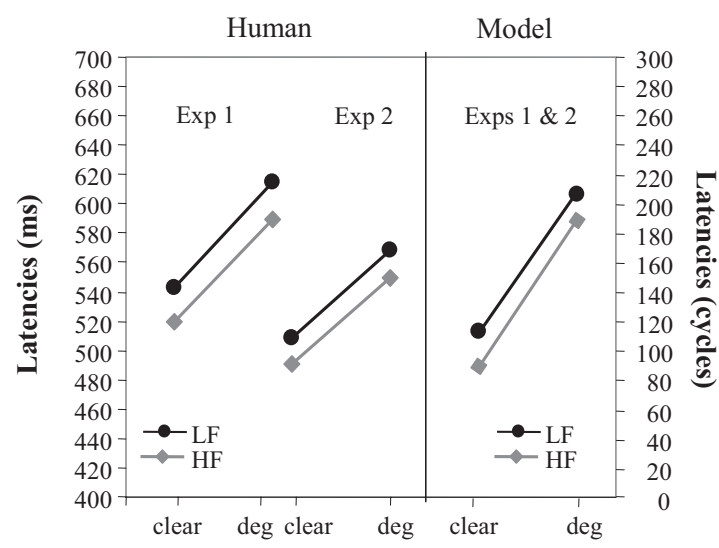

Figure 3. $\mathrm{CDP}+$ simulation of the effects of stimulus quality and word frequency (in the presence of nonwords) obtained in Experiments 1 and 2 of O'Malley and Besner (2008). Note that there is only a single simulation for both experiments, because the experiments used the same items. $\mathrm{CDP}+=$ connectionist dual process model $\mathrm{LF}=$ low frequency; $\mathrm{HF}=$ high frequency; deg $=$ degraded. 


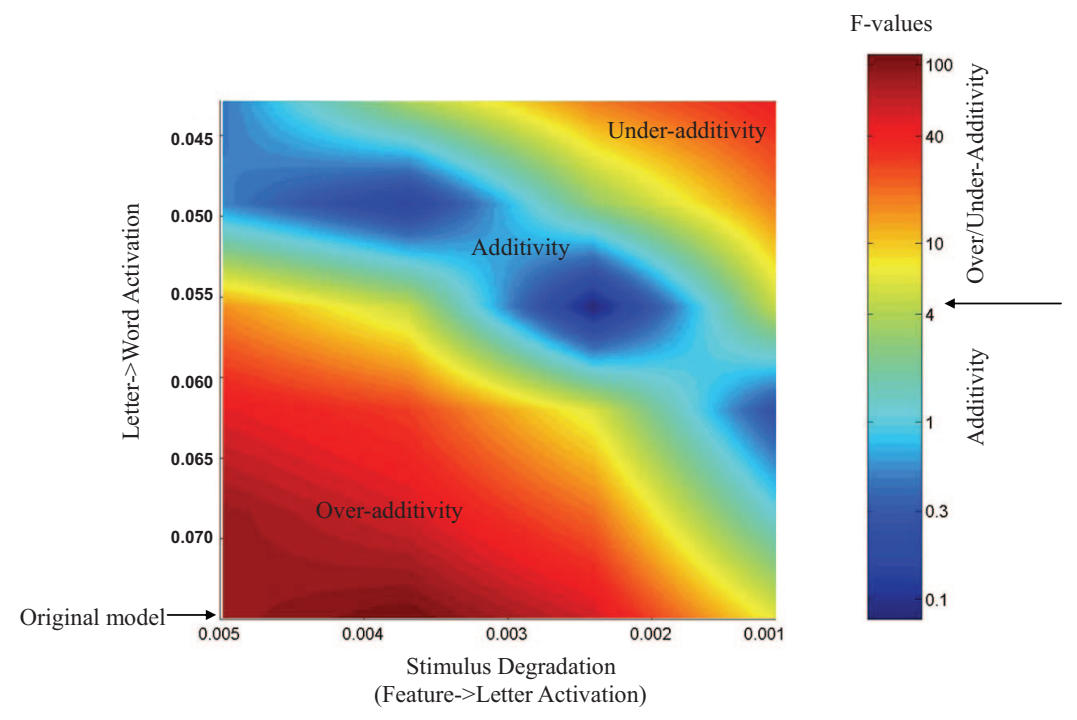

Figure 4. Parametric simulations with $\mathrm{CDP}+$ for the entire set of parameter combinations. Density map represents $F$ values for the interaction between stimulus quality and word frequency on a semilogarithmic scale.

simulations. However, simulating the complexities of strategic adaptation would be a modeling enterprise in its own right and is clearly beyond the scope of the present research.

Coming back to our simulations, the central question is why $\mathrm{CDP}+$ produces additive effects of stimulus quality and frequency in the presence of nonwords. CDP + can simulate additive effects because letter input to the nonlexical route is thresholded. We note that this assumption was not made to account for the effects of stimulus quality; rather, it was made for principled reasons that had nothing to do with these effects. Namely, in CDP + , the graphemic parsing process causes inputs into the sublexical network to be fully activated, even if they are not fully activated when taken from the letter level. This is not the case for DRC, for which the activation of letters in the nonlexical route is identical to that of the letter level in the lexical route. For example, DRC simulations suggest that if the activation buildup at the letter level is slowed due to the presentation of degraded stimuli, nonword length and stimulus quality should have underadditive rather than additive effects; this suggestion is contrary to what is found in human participants. In contrast, the sublexical part of $\mathrm{CDP}+$ essentially operates as if thresholded processing were used at the letter level, and it therefore produces an additive effect of stimulus quality and nonword length for all levels of stimulus degradation. Thresholded processing would certainly fix the DRC model, but the assumption that letters above a specific threshold are given full activation before entering the GPC system seems somewhat post hoc. Our proposal, instead, is that letters above threshold are submitted to a graphemic parsing process that is controlled by focused spatial attention. When inserted in the graphemic buffer, each grapheme is fully active simply because it represents the output of grapheme identification taking place within the attended portion of the letter string.

It is worth noting that there are other ways of simulating additive effects of stimulus quality and word frequency in CDP + . One other way consists of reducing the global activation rate, which is similar to the idea of using input gain to simulate stimulus degradation (Plaut \& Booth, 2000, 2006). The theoretical assumption behind a reduction in global activation is that stimulus degradation would affect the activation rate of all processes. That is, degradation not only would make feature-to-letter activation harder but would affect lexical and nonlexical activation. In this article, we have not chosen this way of simulating the effects, although this would be possible. It is also worth noting that deemphasizing lexical activation will not produce an additive pattern for all parameter values. That is, if we reduce lexical activation even further, the frequency effect would become increasingly small in the degraded condition and thus would produce an underadditive pattern.

This fine balance between over- and underadditivity in CDP+ makes us wonder whether all participants in O'Malley \& Besner (2008) showed a perfectly additive pattern in mixed conditions. Our simulations indeed suggest that one can obtain overadditivity, underadditivity, and additivity, depending on how much lexical activation is reduced and/or nonlexical activation is reinforced. Thus, some participants might show the overadditive pattern, others might show the underadditive pattern, and the average participant would show an additive pattern. This is an important point because computational models simulate item variability, not subject variability (i.e., a given simulation is equivalent to simulation of a single participant). If all participants behave in the same way, this is fine. However, if there was a great deal of intersubject variability in degraded conditions, one would need to take this into account by running simulations for individual participants (for such an approach, see Ziegler et al., 2008; Ziegler, Rey, \& Jacobs, 1998).

In sum, CDP + successfully simulates the multifaceted effects of stimulus quality. In contrast to $\mathrm{DRC}, \mathrm{CDP}+$ provides robust simulations of the effects of stimulus quality on nonword length for a wide range of stimulus degradations. In addition, CDP+ can produce the shift from overadditive to additive effects of stimulus 
quality and word frequency when nonwords are added to the experiment. Whether the latter effect will acquire the status of a benchmark effect for modeling is not up to us to decide, although it is clear that at least one implemented model would pass this benchmark test.

\section{References}

Besner, D., \& Roberts, M. A. (2003). Reading nonwords aloud: Results requiring change in the dual route cascaded model. Psychonomic Bulletin \& Review, 10, 398-404.

Borowsky, R., \& Besner, D. (1993). Visual word recognition: A multistage activation model. Journal of Experimental Psychology: Learning, Memory, and Cognition, 19, 813-840.

Coltheart, M., Rastle, K., Perry, C., Langdon, R., \& Ziegler, J. C. (2001). DRC: A dual route cascaded model of visual word recognition and reading aloud. Psychological Review, 108, 204-256.

Kinoshita, S., \& Lupker, S. J. (2007). Switch costs when reading aloud words and nonwords: Evidence for shifting route emphasis? Psychonomic Bulletin \& Review, 14, 449-454.

O'Malley, S., \& Besner, D. (2008). Reading aloud: Qualitative differences in the relation between stimulus quality and word frequency as a function of context. Journal of Experimental Psychology: Learning, Memory, and Cognition.

O'Malley, S., Reynolds, M. G., \& Besner, D. (2007). Qualitative differences between the joint effects of stimulus quality and word frequency in reading aloud and lexical decision: Extensions to Yap and Balota. Journal of Experimental Psychology: Learning, Memory, and Cognition, 33, 451-458.

Perry, C., Ziegler, J. C., \& Zorzi, M. (2007). Nested incremental modeling in the development of computational theories: The CDP + model of reading aloud. Psychological Review, 114, 273-315.

Plaut, D. C., \& Booth, J. R. (2000). Individual and developmental differences in semantic priming: Empirical and computational support for a single-mechanism account of lexical processing. Psychological Review, 107, 786-823.

Plaut, D. C., \& Booth, J. R. (2006). More modeling but still no stages: Reply to Borowsky and Besner. Psychological Review, 113, 196-200.

Reynolds, M., \& Besner, D. (2004). Neighbourhood density, word frequency, and spelling-sound regularity effects in naming: Similarities and differences between skilled readers and the Dual Route Cascaded Computational model. Canadian Journal of Experimental Psychology, 58, 13-31.

Reynolds, M., \& Besner, D. (2008). Contextual effects on reading aloud: Evidence for pathway control. Journal of Experimental Psychology: Learning, Memory, and Cognition, 34, 50-64.

Yap, M. J., \& Balota, D. A. (2007). Additive and interactive effects on response time distributions in visual word recognition. Journal of Experimental Psychology: Learning, Memory, and Cognition, 33, 274 296.

Zevin, J. D., \& Balota, D. A. (2000). Priming and attentional control of lexical and sublexical pathways during naming. Journal of Experimental Psychology: Learning, Memory, and Cognition, 26, 121-135.

Ziegler, J. C., Castel, C., Pech-Georgel, C., George, F., Alario, F. X., \& Perry, C. (2008). Developmental dyslexia and the Dual Route Model of reading: Simulating individual differences and subtypes. Cognition, 107, 151-178.

Ziegler, J. C., Rey, A., \& Jacobs, A. M. (1998). Simulating individual word identification thresholds and errors in the fragmentation task. Memory \& Cognition, 26, 490-501.

Zorzi, M., Houghton, G., \& Butterworth, B. (1998). Two routes or one in reading aloud? A connectionist dual-process model. Journal of Experimental Psychology: Human Perception and Performance, 24, 11311161.

Received May 13, 2008

Revision received July 18, 2008

Accepted July 19, 2008

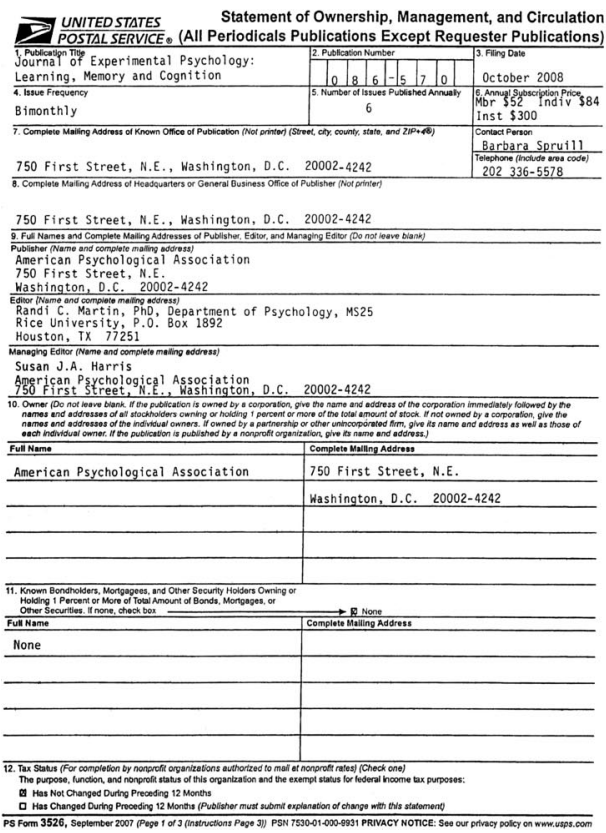

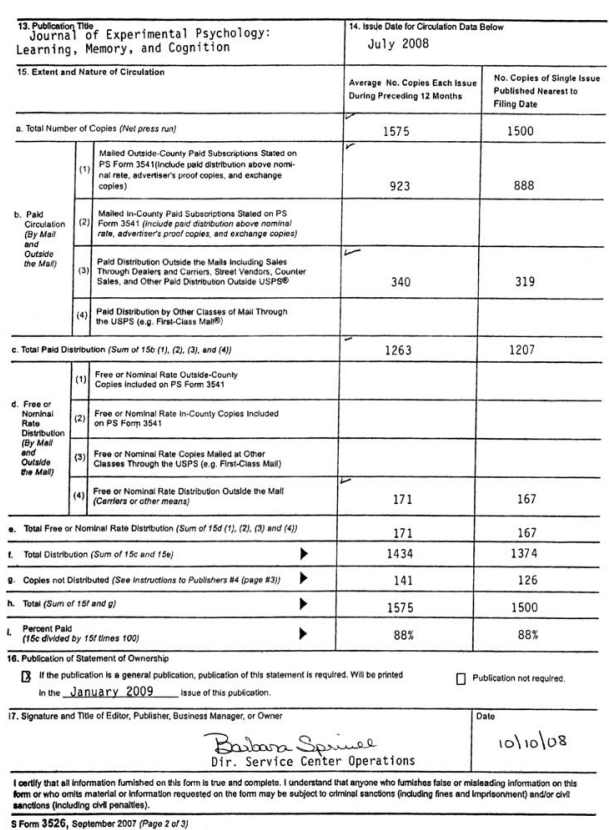

